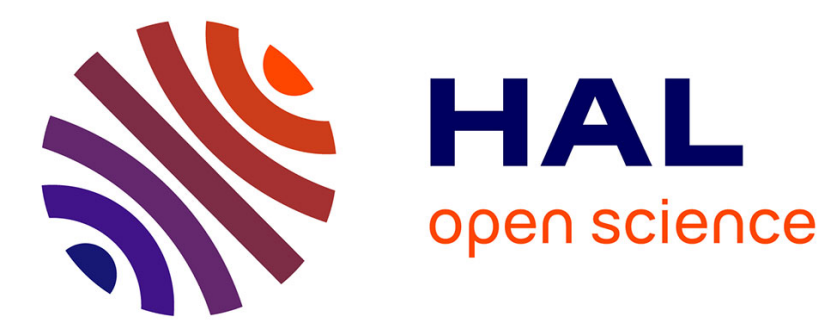

\title{
Observing global states of asynchronous distributed applications
}

\author{
Jean-Michel Hélary
}

\section{To cite this version:}

Jean-Michel Hélary. Observing global states of asynchronous distributed applications. [Research Report] RR-1042, INRIA. 1989. inria-00075516

\section{HAL Id: inria-00075516 https://hal.inria.fr/inria-00075516}

Submitted on 24 May 2006

HAL is a multi-disciplinary open access archive for the deposit and dissemination of scientific research documents, whether they are published or not. The documents may come from teaching and research institutions in France or abroad, or from public or private research centers.
L'archive ouverte pluridisciplinaire HAL, est destinée au dépôt et à la diffusion de documents scientifiques de niveau recherche, publiés ou non, émanant des établissements d'enseignement et de recherche français ou étrangers, des laboratoires publics ou privés. 


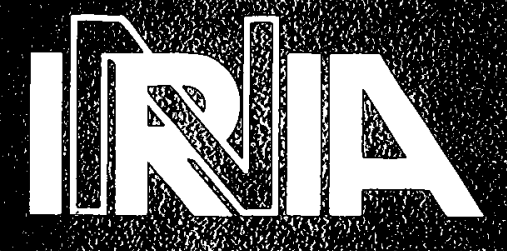

\section{Rapports de Recherche}

UNTI: IVI I. A D D N N ES

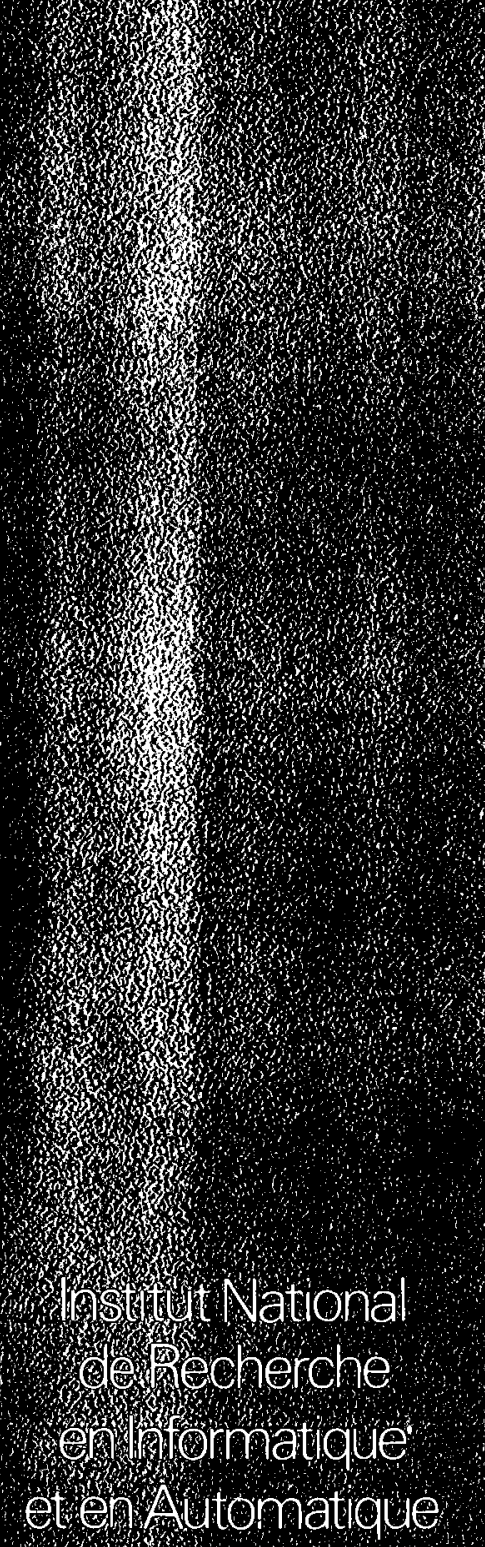

Domaine de Voluceau Rocauencourt BP 105

78153 Le Chesnay Cedex Firance

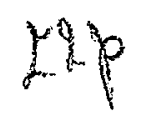

Jean-Michel HELARY 
Campus Universitaire de Beaulieu 35042-RENNES CÉDEX

FRANCE

Téléphone: 99362000

Télex: UNIRISA $950473 \mathrm{~F}$

Télécopie: 99383832

\title{
Observing global states of asynchronous distributed applications.
}

Publication Interne $n^{\circ} 468$ - Avril 1989 - 24 Pages

Observation des états globaux d'applications réparties asynchrones.

\author{
Jean-Michel HELARY \\ IRISA-IFSIC - Campus de Beaulieu-35042 RENNES CEDEX \\ E.mail: helary@irisa.fr
}

\begin{abstract}
Observing global states of an asynchronous distributed application is a difficult task due to arbitrary messages transfer delays. Notion of global states and some of their properties - consistency, being transitless - are precisely stated, and the problem, in both FIFO and non FIFO communication models, is solved in a progressive way : local synchronization allows neighbour processes to record mutually consistent local states, then a sequence of consistent global states is obtained by composition with global wave synchronization; computing some functions over consistent global states becomes easier and an example is displayed (number of messages in transit). Solution generalizes and improves known results, both in FIFO (relaxation of synchronization constraints) and non FIFO (absence of message storing) situations.
\end{abstract}

\section{Résumé}

L'observation d'états globaux d'une application répartie asynchrone est une tâche difficile, notamment à cause du délai arbitraire de transfert des messages. La notion d'états globaux et certaines de leurs propriétés - cohérence, sans transit sont définies avec précision et le problème est résolu de manière progressive, dans les deux modèles de communication FIFO et non FIFO : une synchronisation locale permet aux processus voisins d'enregistrer des élats locaux mutuellement cohérents, puis une séquence d'états globaux cohérents est obtenue par composition avec une synchronisation globale par train de vagues; le calcul de fonctions sur les états globaux cohérents en est facilité et un exemple est présenté (nombre de messages 
en transit). La solution présentée généralise et améliore les résultats déjà connus, auussi bien dans le cas FIFO (relâchement de contraintes de synchronisation) que non FIFO (absence de stockage des messages)

keywords : asynchronism, observation, global state, synchronization, wave sequence, algorithmic composition

\section{Contents}

1 Introduction 3

2 Global state 4

2.1 Distributed application or system ........... 4

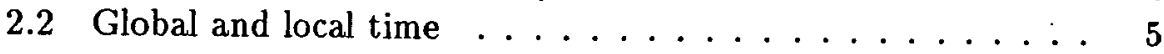

2.3 Global and local states .............. 5

3 Synchronization rules for local mutual consistency 6

3.1 The problem ................ 6

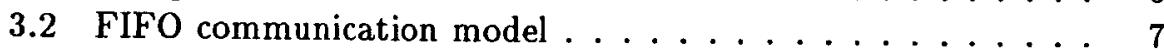

3.3 non-FIFO communication model $\ldots \ldots \ldots \ldots$

3.4 Conclusion $\ldots \ldots \ldots \ldots \ldots \ldots \ldots$

4 Global synchronization for global state consistency 9

4.1 Wave sequences . . . . . . . . . . . . . 10

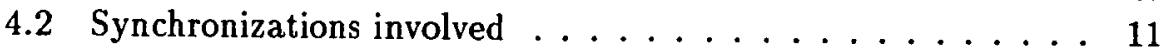

4.2 .1 Case of FIFO model . . . . . . . . . . . . 12

4.2.2 Case of non FIFO communication model . . . . . . 13

5 Computations over consistent global states 16

5.1 FIFO communication . . . . . . . . . . . 16

5.1 .1 Abstract global definitions . . . . . . . . . 16

5.1 .2 Distributed implementation . . . . . . . . . 17

5.2 non FIFO communication ............. 18

5.2.1 Abstract global definitions . . . . . . . . . . 18

5.2 .2 Distributed implementation . . . . . . . . . 19

6 Conclusion $\quad 20$ 


\section{Introduction}

Computing global states of distributed systems or applications (also known as computing snapshots) is a difficult but major problem as previously pointed [CL85, CM88, FGL82, HPR89]. The difficulty is mainly from the lack of global clock and from non determinism inherent to arbitrary transfer delays of messages proper to asynchronism. The interest is yet multiple: on the one hand, it provides solution to a variety of control problems, e.g. detection of stable properties [CM86, Tel86, HJPR87], system recovery after failure [Mor85], etc.; on the other hand, its solution may give prominence to fundamental mechanisms for distributed application control. That's why several interesting results related to this problem have already been obtained. The first published snapshot algorithm was from Chandy and Lamport [CL85]; in this solution the communication channels need to be error-free, unidirectionnal and obey the first-in-first-out property. Synchronization rules use markers as control messages tranmitted on the main computation channels: reception of a marker on a channel is a synchronization event which, due to the FIFO assumption let processes record their local state consistently (we explain these terms in $\S 2$ ). Under the same communication hypotheses, Hélary et al. [HPR89] solved a stronger problem, namely repeated capture of consistent global states, allowing to detect those without messages in transit; the derivation is based upon a principle of algorithm composition, more precisely a phase synchronization (using markers) is combined with a sequential wave synchronization (implemented with a token ring), and the obtained control algorithm is superimposed to the observed application. Among cther proposed solutions, Lai and Yang [LY87] gave an interesting solution dropping out the FIFO assumption; markers aren't used and few synchronization is needed but storage of messages sent and received on every node makes a serious implementation problem. Also free from FIFO assumptions, Mattern [Mat89] gives a nice generalization of Chandy-Lamport's, based upon a generalized virtual time concept.

In this paper, we are interested in deriving snapshot computations based upon general, implementation free wave synchronization and improving previously known solutions in two directions : firstly under FIFO assumptions, marker synchronization constraints are weakened; secondly, we drop out the FIFO model, assuming only that channels are reliable, and unlike Lai and Yang's, only local counters storage, periodically reset to zero, is needed; in that sense, our result is similar to Mattern's one, but unlike the latter we achieve termination of a snapshot computation independantly of observed 
application behaviour, and our derivation and implementation are different.

In $\S 2$ we define precisely the notions of distributed application, its global state and some of their properties (consistency, no transit, etc.). In $\S 3$ we set synchronization rules allowing processes to record their local states consistently according to communication hypotheses; these rules define a distributed control algorithm superimposed to the underlying observed application, whereas $\$ 4$ shows how a global synchronization technique - namely wave sequence - can be used to implement some computations on the set of recorded local states. An example of such a computation is given in $\S 5$ : number of intransit messages in a global state, allowing detection of global states without messages in transit, both in reliable FIFO and non-FIFO communication models.

\section{Global state}

\subsection{Distributed application or system}

Application (or system) to be observed consists of a set of $n$ computing processes $X=\left\{P_{1}, P_{2}, \ldots, P_{n}\right\}$, interacting through message exchange only; each process is located on a node, named a computing node in the following; messages circulate along a set $U \subseteq X \mathbf{x} X$ of communication channels ${ }^{1}$. We shall denote by $c_{i j}$ the channel connecting $P_{i}$ to $P_{j}$.

Several communication models can be defined:

- either synchronous, e.g. :

rendez-vous (instant communication)

bounded delay (known maximum message transfer delay)

- or asynchronous, e.g. :

reliable FIFO (finite but impredictable message transfer delay : each channel acts like an unbounded FIFO buffer)

bounded FIFO (like FIFO but with limited amount of storage : each channel acts like a bounded buffer and thus can lost messages or cause sender's blocking in case of full buffer)

reliable non FIFO (messages can be desequenced but neither lost nor duplicated nor altered)

\footnotetext{
${ }^{1}$ Channels are assumed to be logically directed but physically bidirectionnal
} 


\subsection{Global and local time}

Whatever the model, some common features concerning time are to be noticed :

- There is no global clock, i.e. there is no concrete global time shared by the $n$ processes,

- on the other hand, we will assume existence of an abstract global time (for purpose of reasonning only); this abstract time obeys to the causality principle : for any message $m$, emission precedes reception :

$$
e m(m)<_{\text {agt }} \operatorname{rec}(m)
$$

where $<_{a g t}$ is the precedence relation in this abstract global time,

- each process is endowed with a local clock; local events (message sending or receiving and internal events) are sequential and totally ordered in the local time; this ordering is compatible with the abstract global time. In the following we shall use the term time for local time.

Several well-known implementations of such an abstract global time exist, e.g. [Lam78, Mat89]

\subsection{Global and local states}

For each process $P_{i} \in X$, the local state of $P_{i}$ at a given time is defined by the local context of the distributed application (projection onto $P_{i}$ of the global context). A global state is a set $G S=\left\{L S_{1}, L S_{2}, \ldots, L S_{n}\right\}$ containing processes local states. We shall denote by $\tau\left(L S_{i}\right)\left(\tau_{i}\right.$ in brief) the time when $P_{i}$ has recorded its local state $L S_{i}$. For a message $m_{i j}$, sent from $P_{i}$ to $P_{j}$ (along channel $c_{i j}$ ) we shall say that :

- its emission belongs to $L S_{i}$ iff $e m\left(m_{i j}\right)<\tau\left(L S_{i}\right)$

- its reception belongs to $L S_{j}$ iff $\operatorname{rec}\left(m_{i j}\right)<\tau\left(L S_{j}\right)$ 
For a message $m_{i j}$ and a pair of local states $\left(L S_{i}, L S_{j}\right)$, we denote the four possible configurations in the following way:

(i) $\quad e m\left(m_{i j}\right) \in L S_{i} \wedge \operatorname{rec}\left(m_{i j}\right) \in L S_{j} \rightarrow$ consumed message

(ii) $\quad e m\left(m_{i j}\right) \in L S_{i} \wedge \operatorname{rec}\left(m_{i j}\right) \notin L S_{j} \rightarrow$ intransit message $m_{i j} \in \operatorname{transit}\left(L S_{i}, L S_{j}\right)$

(iii) $e m\left(m_{i j}\right) \notin L S_{i} \wedge \operatorname{rec}\left(m_{i j}\right) \in L S_{j} \rightarrow$ inconsistent message

(iv) $\quad e m\left(m_{i j}\right) \notin L S_{i} \wedge \operatorname{rec}\left(m_{i j}\right) \notin L S_{j} \rightarrow$ inexistent message $m_{i j} \in$ inexistent $\left(L S_{i}, L S_{j}\right)$

The state of a channel $c_{i j}$ with respect to a pair of local states $\left(L S_{i}, e l_{j}\right)$ can be defined as the set of messages

$$
C S_{i j}=\operatorname{transit}\left(L S_{i}, L S_{j}\right) \bigcup \text { inconsistent }\left(L S_{i}, L S_{j}\right)
$$

Stress mut be laid on the fact that, in a distributed system, only processes local states are (locally) observable at a given time; the state of a given channel is defined formally, from both states of its end processes.

We now give the following definitions concerning global states :

Definition 1. Global state $G S=\left\{L S_{1}, L S_{2}, \ldots, L S_{n}\right\}$ is consistent if, and only if,

$$
\forall i \forall j \text { inconsistent }\left(L S_{i}, L S_{j}\right)=\emptyset
$$

Definition 2. Global state $G S=\left\{L S_{1}, L S_{2}, \ldots, L S_{n}\right\}$ is transitless if, and only if,

$$
\forall i \forall j \operatorname{transit}\left(L S_{i}, L S_{j}\right)=\emptyset
$$

Definition 3. A global state is strongly consistent if, and only if, it is consistent and transitless [HPR89]

This last property means that, for any pair $L S_{i}, L S_{j}$, a message $m_{i j}$ is received in $L S_{j}$ if, and only if, it is sent in $L S_{i}$.

\section{Synchronization rules for local mutual consis- tency}

\subsection{The problem}

When a process $P_{i}$ decides to record a local state $L S_{i}$ it is necessary that it proceeds in cooperation with its neighbours, more precisely with processes 
$P_{j}$ such that $\left(P_{j}, P_{i}\right) \in U$; in fact, inconsistent messages with respect to the pair $\left(L S_{j}, L S_{i}\right)$ should be avoided, since they are messages having been received but not yet sent (in the global state $G S=\left\{\ldots, L S_{j}, \ldots, L S_{i}, \ldots\right\}$ ). The problem to be considered here can be stated more precisely : consider a pair of connected processes $\left(P_{j}, P_{i}\right) \in U$, each of them decides to record its local state respectively at times $\tau_{j}$ and $\tau_{i}$. How to synchronise $\tau_{j}$ and $\tau_{i}$ in order to have inconsitent $\left(L S_{j}, L S_{i}\right)=\emptyset$ ?

The answer depends on the communication model; we will not be concerned by synchronous models (rendez-vous, bounded delays) since problem here is obvious; we'll focus, in what follows, on two asynchronous models : FIFO (unbounded or not) and non FIFO. In order to implement a synchronization control which doesn't disturb the observed application, we will assume that on every computing node, there exists a control process $C T L$ which performs the following tasks (let $P$ be the application process associated to $C T L$ on the same node):

i) monitor message sending and receiving on the account of $P$ (this action must neither create nor modify nor desequence messages $:$ it should be limited to observation and synchronisation),

ii) handle control messages, transparent to $P$,

iii) record local states on the account of $P$.

\subsection{FIFO communication model}

This model is characterized by the following property : let $m$ and $m$ ' two messages circulating over the same channel; then

$$
\epsilon m(m)<e m\left(m^{\prime}\right) \Rightarrow\left(\operatorname{rec}(m)<\operatorname{rec}\left(m^{\prime}\right) \vee \operatorname{rec}(m)=\infty\right)
$$

(The second term handles with loss of messages in non reliable or bounded buffer models). The set of messages sent over a given channel has an ordered list structure which leads to use control messages acting like end-of-(sub)file markers.

Let two processes $\left(P_{j}, P_{i}\right) \in U$ having recorded local states $L S_{j}, L S_{i}$ at respective times $\tau_{j}, \tau_{i}$. Let $m_{j i}$ a message sent over chanel $c_{j i}$ and $\tau_{j}<$ $e m\left(m_{j i}\right)$. To insure inconsistent $\left(L S_{j}, L S_{i}\right)=\emptyset$ it is sufficient that $\tau_{i}<$ $\operatorname{rec}\left(m_{j i}\right)$. Suppose $C T L_{j}$ sends a control message $m a r k_{j i}$ over $c_{j i}$ at time $\tau_{j}$. We have then $e m\left(\operatorname{mark}_{j i}\right)=\tau_{j}<e m\left(m_{j i}\right)$ whence, from FIFO assumption: $\operatorname{rec}\left(\operatorname{mark}_{j i}\right)<\operatorname{rec}\left(m_{j i}\right) ;$ from this follows that the relation $\tau_{i} \leq \operatorname{rec}\left(\operatorname{mark}_{j i}\right)$ 


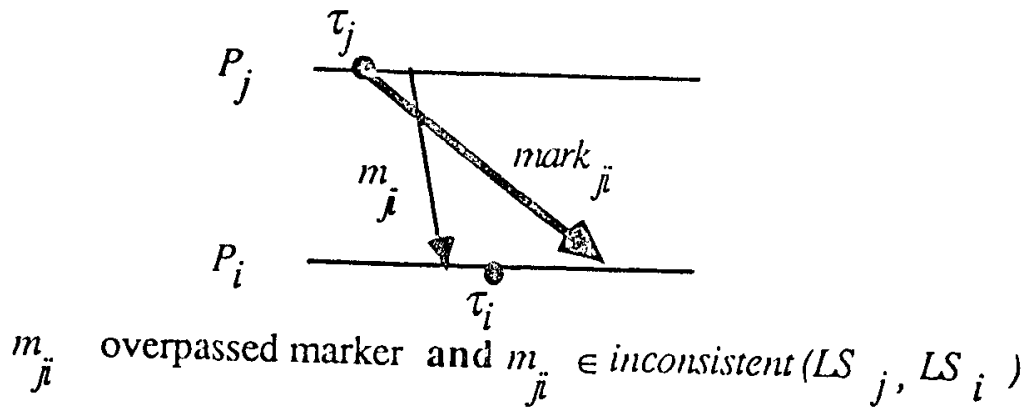

Figure 1: non-FIFO communication

insures the desired consistency property. We have obtained the following lemma :

Lemma 3.1. Let mark $k_{j i}$ a control message sent over $c_{j i}$ when $C T L_{j}$ records the local state $L S_{j}$ of $P_{j}$. Then, for every local state $L S_{i}$ of process $P_{i}$ recorded by $C T L_{i}$ not later t?a.s the reception of $m a r k_{j i}$, the property inconsistent $\left(L S_{j}, L S_{i}\right)=0$ iolis.

Remark. This result remains true in case of application message loss, since the relation $\operatorname{rec}\left(m_{j i}\right)=\infty$ impl: $\operatorname{rec}\left(n_{j i}\right)>\tau_{i}$. On the other hand, loss of control message mark $k_{j i}$ makcs the lemma meaningless since the event rec $\left(\operatorname{mark}_{j i}\right)$ never happens!

\section{3 non-FIFO communication model}

In that case, the set of messages sent ovcr a given channel is no more totally ordered and using markers is no more sufficient to insure the desired consistency property. Figure 1 shows that $L S_{i}$ 's recording occurs too late with respect to $L S_{j}$ 's recording, despite the marker's synchronization. Time $\tau_{j}$ has to be delayed in order that messages $m_{j i}$ sent after $\tau_{j}$ cannot reach $P_{i}$ before $\tau_{i}$. To this end, an acknowledgement technique can be used : when $C T L_{j}$ decides to record $P_{j}$ 's local state $L S_{j}$, it sends a marker $m a r k_{j i}$ over channel $c_{j i}$; when $C T L_{i}$ receives this marker, it sends back an acknowledgement message $a c k_{i j}$ to $C T L j$. The latter cannot record $L S_{j}$ before the reception of this ack (figure 2). Let's show that this technique leads to the following result :

Lem ma 3.2. Let mark $k_{j i}$ a control message sent over $c_{j i}$ by $C T L j$ and $a c k_{i j}$ its acknowledgement of receipt. For any local state $L S_{j}$ such that 


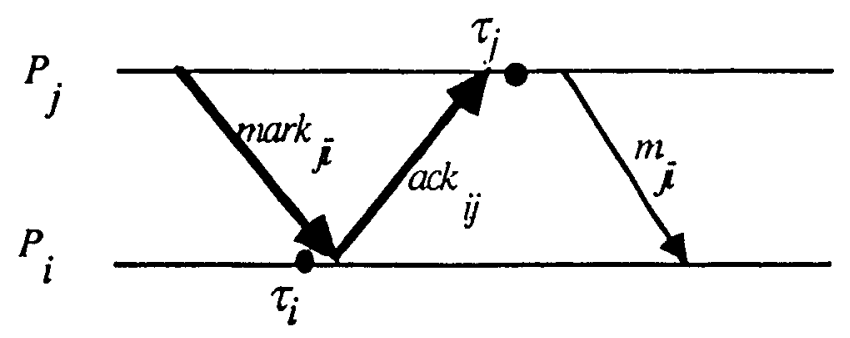

Figure 2: Acknowledgement technique

$\tau\left(L S_{j}\right) \geq \operatorname{rec}\left(a c k_{i j}\right)$ and every local state $L S_{i}$ such that $\tau\left(L S_{i}\right) \leq$ $\operatorname{rec}\left(\operatorname{marq}_{j i}\right)$ the property inconsistent $\left(L S_{j}, L S_{i}\right)$ holds.

Proof. Let $m_{j i}$ such that $e m\left(m_{j i}\right)>\tau_{j}$. The following relations hold :

$$
\begin{aligned}
\operatorname{rec}\left(m_{j i}\right) & >_{a g t} e m\left(m_{j i}\right) & & \text { (causality) } \\
& >\tau_{j} & & \text { (by construction) } \\
& \geq \operatorname{rec}\left(a c q_{i j}\right) & & \text { (hypothese) } \\
& >_{a g t} e m\left(a c q_{j i}\right) & & \text { (causality) } \\
& =\operatorname{rec}\left(\operatorname{marq_{ji}}\right) & & \text { (by ack definition) } \\
& \geq \tau_{i} & & \text { (hypothese) }
\end{aligned}
$$

\subsection{Conclusion}

This section was devoted to local synchronization between adjacent nodes in order to avoid inconsistant messages on incoming channels. We didn't handle the problem of deadlock occurence which could result from delays involved in these local synchronizations; the next section will be devoted to global synchronization techniques, leading to consistent and deadlock free global states recording.

\section{Global synchronization for global state consis- tency}

This section deals with the following problem : each process of the observed application has to record a sequence of local states $\left(L S_{i}^{(\mu)}\right)_{\mu \in N}$ such 
that $\forall \mu \forall\left(P_{i}, P_{j}\right) \in U$ : inconsistent $\left(L S_{i}^{(\mu)}, L S_{j}^{(\mu)}\right)=\emptyset$, in other words : $\forall \mu G S(\mu)=\left\{L S_{1}^{(\mu)}, L S_{2}^{(\mu)}, \ldots, L S_{n}^{(\mu)}\right\}$ is a consistent global state. This problem will be treated here by composing two distributed algorithmic techniques :

1. Markers (resp. markers and acknowledgements) synchronization depending whether the communication model is FIFO or not, as seen in the preceeding section,

2. Wave sequence synchronization.

The first synchronization insures local mutual consistency for every pair $\left(L S_{i}, L S_{j}\right)$ whereas the second one insures global state consistency and controls transition from a global state computation $G S(\mu)$ to the next one $G S(\mu+1)$; moreover, data carried over by waves make possible to perform some computations on recorded global states such that, for instance, " $G S(\mu)$ is transitless,..."

\subsection{Wave sequences}

A wave sequence is an abstract control scheme expressing the concept of global distributed iteration [HR89]. A wave [Sch85] is a control flow visiting every process in the system once, and only once, and allowing at least one process, predetermined or not, to know when this traversal is over. A wave defines the following events :

$\forall P_{i}$ visit $(i) \equiv$ process $P_{i}$ is visited by the wave

return $\equiv \forall P_{i}$ visit $(i)$ happened

The set of visits is called a traversal.

As data flow, a wave has two functions : it broadcasts a datum DIFF and collects a datum COLL. $P_{i}$ from each process $P_{i}$ (these functions are performed on each process upon the visit); moreover, data collected allow to perform computation COLL $=f\left(C O L L . P_{1}, C O L L . P_{2}, \ldots, C O L L . P_{n}\right)$. The logic associated to a wave is thus the following :

$\% \forall i P_{i}$ knows $C O L L . P_{i} \wedge$

$\exists I \subseteq X, I \neq \emptyset \wedge(P \in I \Rightarrow P$ knows $D I F F) \%$

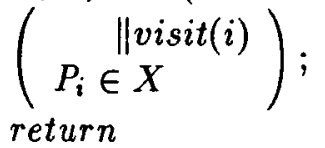


$\% \forall i P_{i}$ knows $D I F F \wedge$

$\exists J \subseteq X, J \neq \emptyset \wedge\left(P \in J \Rightarrow P\right.$ knows $\left.f\left(C O L L . P_{1}, \ldots, C O L L . P_{n}\right)\right) \%$

Moreover, following temporal relations hold (with straightforward notations) :

$$
\forall \mu(\forall i \operatorname{visit}(i, \mu))<_{\text {agt }} \operatorname{return}(\mu)<_{\text {agt }}(\forall i \operatorname{visit}(i, \mu+1))
$$

A wave sequence ensures a strong global synchronization since the event return $(\mu)$ separates the two sets of events

$$
\left\{\operatorname{visit}(i, \mu) \mid P_{i} \in X\right\} \text { and }\left\{\operatorname{visit}(i, \mu+1) \mid P_{i} \in X\right\}
$$

In other words, a global state exists for which all the events of the first set and none of the second set occured [Tel86].

Wave sequences may be implemented by various traversal structures : directed or undirected spanning trees, rings, etc.. They lead to sequential or parallel structuration of wave events, centralized or distributed return control, and so on [HR88b, Tel86]

\subsection{Synchronizations involved}

Wave sequence will be useful to :

- Global synchronization of local states recordings; global state $G S(\mu)$ will be recorded along with wave $\mu$,

- collection (resp diffusion) of informations related to global states recorded by the current wave (resp. previous wave).

Thus the first synchronization rule can be stated as follows :

R1) A controller $C T L_{i}$ records local state $L S_{i}^{(\mu)}$ of process $P_{i}$ upon the event visit $(i, \mu)$ (This event can possibly be delayed by $C T L_{i}$ until the actual recording of $\left.L S_{i}^{(\mu)}\right)$.

We now state and proof rules to insure consistency of global state $G S(\mu)$, according to the communication model 


\subsubsection{Case of FIFO model}

R2) When $C T L_{i}$ records $L S_{i}^{(\mu)}$, it sends a marker $\operatorname{mark}_{i j}(\mu)$ over each outgoing channel $c_{i j}$

R3) (freezing rule) If $C T L_{i}$ receives the marker $\operatorname{mark}_{j i}(\mu)$ on the incoming channel $c_{j i}$ before the occurence of event visit $(i, \mu)$, it prevents process $P_{i}$ from receiving computations messages or markers on that channel until local state $L S_{i}^{(\mu)}$ is recorded.

We state the main result of this subsection :

Theorem 4.1. If communications obey FIFO hypotheses and don't loose control messages (markers and wave implementation messages), rules R1) to R3) imply

$$
\forall \mu G S(\mu) \text { is consistent }
$$

Proof. Let $P_{i}$ be a process, $\tau_{i}(\mu)$ the time when $C T L_{i}$ records $L S_{i}^{(\mu)}$ and $\sigma_{j i}(\mu)$ the time when $C T L_{i}$ receives marker mark $_{j i}$ on an incoming channel $c_{j i}$ (sent by $C T L_{j}$ at time $\tau_{j}(\mu)$ ). By rule R3) : $\tau_{i}(\mu) \leq \sigma_{j i}(\mu)$ whence, from lemma 3.1 : inconsistent $\left(L S_{j}^{(\mu)}, L S_{i}^{(\mu)}\right)=\emptyset \square$

Remark on the absence of deadlock. This point is straightforward since record of local state depends only of wave progression.

Remark on markers and waves identification. Due to global wave sequence synchronization, any marker $\operatorname{mark}_{j i}(\nu)$ arriving on incoming channel $c_{j i}$ before time $\tau_{i}(\mu)$ has a number $\nu$ less or equal to $\mu$ (in the case $\nu=\mu$, and only in that case, channel $c_{j i}$ is "frozen" until time $\left.\tau_{i}(\mu)\right)$. Indeed, $\operatorname{mark}_{j i}(\mu+1)$ is sent at time $\tau_{j}(\mu+1)$; but, from abstract global time relations due to wave sequence synchronization : $\tau_{j}(\mu+1)>_{a g t} \tau_{i}(\mu)$ and thus $\sigma_{j i}(\mu+1)>\tau_{i}(\mu)$. This fact is noteworthy as far as implementation is concerned : markers and wave numbers needn't to be explicit. Each controller $C T L_{i}$ handles a local counter array $C_{i}$, indexed by incoming channels, such that :

$\forall j C_{i}[j] \leftarrow C_{i}[j]+1$ upon recording of a new local state

$C_{i}[j] \leftarrow C_{i}[j]-1$ upon reception of a new marker on $c_{j i}$

If $C_{i}[j]$ is nul when a marker is received on $c_{j i}$ then this channel is "frozen" until the next local state recording (at this time the value $C_{i}[j]$ is kept unchanged since it is increased then immediatly decreased by one). 


\subsubsection{Case of non FIFO communication model}

R'2) $C T L_{i}$ cannot record local state $L S_{i}^{(\mu)}$ until it has received, on each incoming channel $c_{j i}$, the acknowledgemenet $a c k_{j i}(\mu-1)$ related to marker $\operatorname{mark}_{i j}(\mu-1)$ (prior to the first visit we assume that every process sends an ack $(0)$ message on every outgoing channel),

R'3) When $C T L_{i}$ records local state $L S_{i}^{(\mu)}$, it sends a marker $\operatorname{mark} k_{i j}(\mu)$ over each outgoing channel $c_{i j}$

R'4) (freezing rule) Once $C T L_{i}$ received the marker $\operatorname{mark}_{j i}(\mu-1)$ on the incoming channel $c_{j i}$ it prevents process $P_{i}$ from receiving computations messages or markers on that channel until local state $L S_{i}^{(\mu)}$ is recorded.

We state now the main result of this subsection :

Theorem 4.2. If communications are non FIFO and don't loose control messages (markers, acknowledgements and wave implementation messages), rules R1) and R'2 to R'4) imply

$$
\forall \mu G S(\mu) \text { is consistent }
$$

Proof. Let $P_{i}$ and $P_{j}$ two processes such that $\left(P_{i}, P_{j}\right) \in U$ and $m_{i j}$ a message such that $e m\left(m_{i j}\right)>\tau_{i}(\mu)$. We must show that $\operatorname{rec}\left(m_{i j}\right)>\tau_{j}(\mu)$. Let us denote $\alpha_{j i}(\mu-1)$ the time when $C T L_{i}$ received the acknowledgement $a c k_{j i}(\mu-1)$ related to marker $\operatorname{mark}_{i j}(\mu-1)$. By construction, rule R'2 and causality, the following relations hold :

$$
e m\left(m_{i j}\right)>\tau_{i}(\mu) \geq \alpha_{j i}(\mu-1)>_{a g t} \sigma_{i j}(\mu-1)
$$

thus, on process $P_{j}: \operatorname{rec}\left(m_{i j}>\sigma_{i j}(\mu-1)\right.$ (causality).

Two situations may occur on channel $c_{i j}$ :

i) $\operatorname{mark}_{i j}(\mu-1)$ is received by $C T L_{j}$ before time $\tau_{j}(\mu)$ (it is not overpassed by $a c q_{i j}(\mu-1)$ : fig 3$)$. In that case, by rule $\mathbf{R}^{\prime} 4$ receptions on channel $c_{i j}$ are "frozen" by $C T L_{j}$ in on the time interval $\left[\sigma_{i j}(\mu-1), \tau_{j}(\mu)\right]$ whence $\operatorname{rec}\left(m_{i j}\right)>\tau_{j}(\mu)$

ii) $\operatorname{mark}_{i j}(\mu-1)$ is received by $C T L_{j}$ after time $\tau_{j}(\mu)$ (it is overpassed by $a c q_{i j}(\mu-1)$ : fig 4$)$. In that case, by causality, construction and rule R'2), the following relations hold :

$$
\operatorname{rec}\left(m_{i j}\right)>_{a g t} e m\left(m_{i j}\right)>\tau_{i}(\mu) \geq \alpha_{j i}(\mu-1)>_{a g t} \sigma_{i j}(\mu-1)>\tau_{j}(\mu)
$$




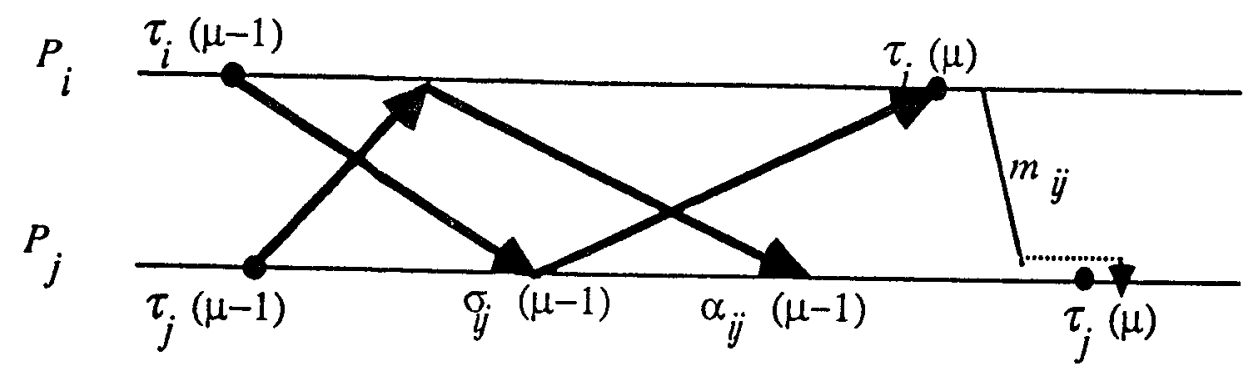

Figure 3: situation i)

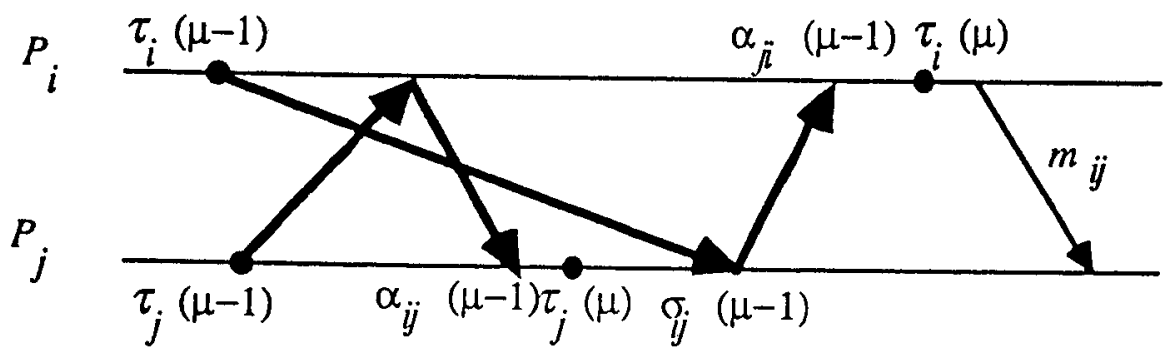

Figure 4: situation ii) 


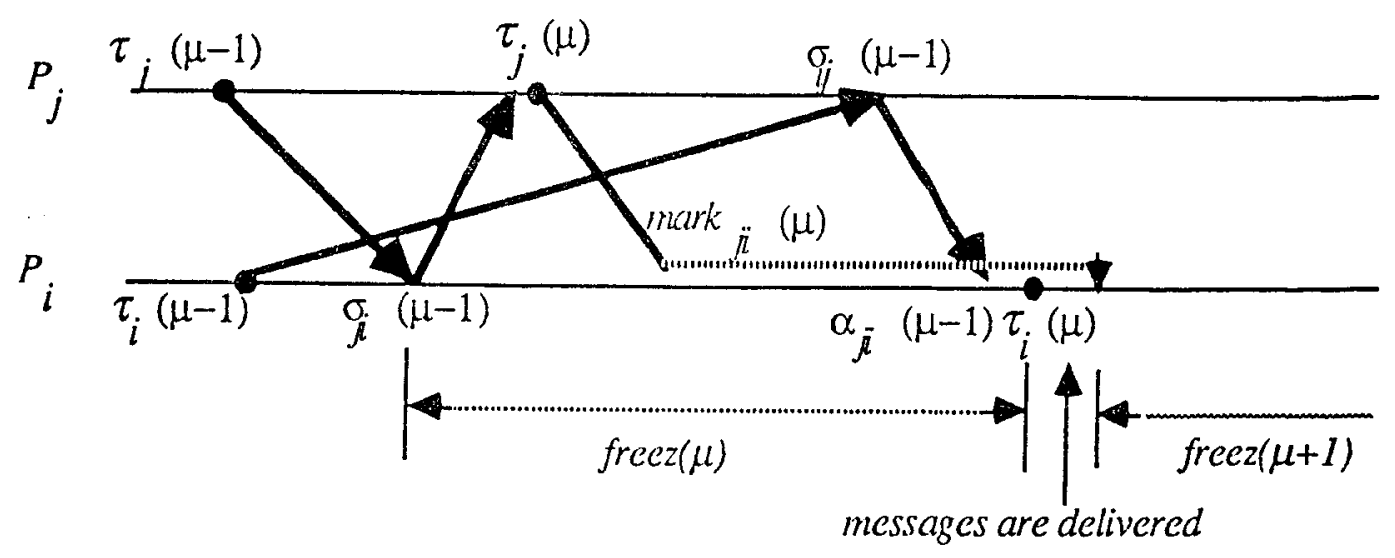

Figure 5: non overlapping of freezing periods

Remark on the absence of deadlock. Two asuumptions have been made :

i) When controllers start to observe underlying application, they send an $a c k(0)$ message on every outgoing channel (prior to the first wave visit)

ii) Control messages have a finite transfer delay (they cannot be lost)

We show, by induction on the wave number, that $\forall \mu$ event return $(\mu)$ eventually occurs.

- From i) and ii), every controller $C T L_{i}$ receives messages $a c k_{j i}(0)$ on each of its incoming channels $c_{j i}$ after a finite time, thus $L S_{i}^{(1)}$ is eventually recorded whence wave 1 returns after a finite time

- Suppose return $(\mu-1)$ has occured. Each controller $C T L_{i}$ has recorded $L S_{i}^{(\mu-1)}$, and at that time, sent $\operatorname{mark}_{i j}(\mu-1)$ over outgoing channel $c_{i j}$; thus, from ii), it will receive $a c q_{j i}(\mu-1)$ a finite time later, whence $L S_{i}^{(\mu)}$ recording cannot be indefinitely delayed. Thus $\operatorname{return}(\mu)$ eventually occurs $\square$

Remark on the non overlapping of freezing periods. To make sure that messages kept over by a controller $C T L_{i}$ during "freezing" periods will be delivered to process $P_{i}$ within a finite time, it is necessary (and sufficient) that freezing periods don't overlap. Let's denote by freez $(\mu)=$ $\left[\sigma_{j i}(\mu-1), \tau_{i}(\mu)\right]$ the eventual freezing period on channel $c_{j i}$ preceeding the local state $L S_{i}^{(\mu)}$ recording (we will assume that it's not empty, otherwise the question is meaningless; see fig 5) The freezing period freez $(\mu+1)$, if not empty, is defined by the interval $\left[\sigma_{j i}(\mu), \tau_{i}(\mu+1)\right]$. But $\sigma_{j i}(\mu)>$ 
$\sigma_{j i}(\mu-1)$, in other words $\operatorname{mark}_{j i}(\mu)$ reaches $C T L_{i}$ during or after the interval freez $(\mu)$, thus $\sigma_{j i}(\mu)>r_{i}(\mu)$ holds. When $C T L_{i}$ records $L S_{i}^{\mu}$, all messages which arrived during $f r e e z(\mu)$ are delivered to $P_{i}$, afterwards, if marker $\operatorname{mark}_{j i}(\mu)$ was among these messages, freezing period freez $(\mu+1)$ begins. Moreover, no marker $\operatorname{mark}_{j i}(\nu), \nu \geq \mu+1$ reached $C T L_{i}$ during freez( $\mu$ ) since $\operatorname{mark}(\mu+1)$ is sent at time $\tau_{j}(\mu+1)>_{a g t} \tau_{i}(\mu) \square$

\section{Computations over consistent global states}

This section adresses the problem of computing functions defined over consistent global states recorded through a wave. The following example will be considered : compute the number card(intransit $(G S(\mu)))$ of intransit messages in the global state $G S(\mu)$ (for any $\mu$ ). One of the applications of this computation is, for instance : is the recorded global state strongly consistent? The two communication models : reliable unbounded FIFO and reliable unbounded non FIFO will be considered. In the following, we say that a message $m_{j i}$ is sent (resp. received) in $G S(\mu)$ if, and only if : $\tau_{j}(\mu-1)<e m\left(m_{j i}\right)<\tau_{j}(\mu)\left(\operatorname{resp} . \tau_{i}(\mu-1)<\operatorname{rec}\left(m_{j i}\right)<\tau_{i}(\mu)\right)$, in other words, its emission (resp. reception) belongs to $G S(\mu)$ but not to $G S(\mu-1)$.

\subsection{FIFO communication}

\subsubsection{Abstract global definitions}

With every wave number $\mu$ and any node identity $i$ let's associate the following sets :

$$
\begin{aligned}
E_{i}(\mu) & =\bigcup_{j \neq i}\left\{m_{j i} \mid \operatorname{em}\left(m_{j i}\right)<\tau_{j}(\mu)\right\} \\
R_{i}(\mu) & =\bigcup_{j \neq i}\left\{m_{j i} \mid \operatorname{rec}\left(m_{j i}\right)<\tau_{i}(\mu)\right\} \\
T_{i}(\mu) & =\bigcup_{j \neq i}\left\{m_{j i} \mid \operatorname{em}\left(m_{j i}\right)<\tau_{j}(\mu) \wedge \operatorname{rec}\left(m_{j i}\right)>\tau_{i}(\mu)\right\}
\end{aligned}
$$

Note that $T_{i}(\mu)$ is the set of intransit messages towards $P_{i}$ in global state $G S(\mu)$. We have the following result :

Theorem 5.1. If $G S(\mu)$ is consistent, then $T_{i}(\mu)=E_{i}(\mu)-R_{i}(\mu)$ 
Proof. From $G S(\mu)$ consistency follows $R_{i}(\mu) \subseteq E_{i}(\mu)$; moreover, by construction, $T_{i}(\mu) \subseteq E_{i}(\mu)$, whence $R_{i}(\mu) \cup T_{i}(\mu) \subseteq E_{i}(\mu)$. Conversely relation $E_{i}(\mu) \subseteq R_{i}(\mu) \bigcup T_{i}(\mu)$ clearly holds, whence $E_{i}(\mu)=R_{i}(\mu) \bigcup T_{i}(\mu)$ and since $R_{i}(\mu) \cap T_{i}(\mu)=\emptyset$, it follows that $R_{i}(\mu)$ and $T_{i}(\mu)$ are a partition of $E_{i}(\mu)$, in other words, $T_{i}(\mu)=E_{i}(\mu)-R_{i}(\mu) \square$

Consider now the global abstract counter vector $M T(\mu)$ defined by

$$
M T(\mu)[i]=\sum_{j \neq i} \operatorname{card}\left(E_{i}(\mu)\right)-\sum_{j \neq i} \operatorname{card}\left(R_{i}(\mu)\right)
$$

The following corollaries are immediate from theorem 5.1 :

Corollary 5.1. If $G S(\mu)$ is consistent, then $\forall i M T(\mu)[i]=\sharp$ messages intransit towards $P_{i}$ in $G S(\mu)$.

Corollary 5.2. If $G S(\mu)$ is consistent, then it is transitless if, and only if, $M T(\mu) \equiv 0$

\subsubsection{Distributed implementation}

The distributed computation of counters $M T(\mu)$ will be implemented using the wave sequence through which $G S(\mu)$ is recorded : informations $D I F F$ and $\forall i C O L L . P_{i}$ have to be carried over by waves in order to maintain the following invariant :

Wave invariant. (When wave $\mu$ returns : $\operatorname{COLL}(\mu)=M T(\mu)$ )

$$
\wedge D I F F(\mu+1)=\operatorname{COLL}(\mu)
$$

Progression. By definition :

$$
\begin{aligned}
\forall \mu \forall i M T(\mu)[i]= & M T(\mu-1)[i] \\
& +\sum_{j \neq i} \operatorname{card}\left\{m_{j i} \mid \tau_{j}(\mu-1)<\operatorname{em}\left(m_{j i}<\tau_{j}(\mu)\right\}\right. \\
& -\sum_{j \neq i} \operatorname{card}\left\{m_{j i} \mid \tau_{i}(\mu-1)<\operatorname{rec}\left(m_{j i}<\tau_{i}(\mu)\right\}\right. \\
= & M T(\mu-1)[i] \\
& +\sum_{j \neq i} \operatorname{card}\left\{m_{j i} \mid m_{j i} \text { sent in } G S(\mu)\right\} \\
& -\sum_{j \neq i} \operatorname{card}\left\{m_{j i} \mid m_{j i} \text { received in } G S(\mu)\right\}
\end{aligned}
$$

Line (a) is nothing else, from the invariant, than $D I F F(\mu)[i]$. Each of lines (b) and (c) can be computed from local counters : each controller $C T L_{i}$ is endowed with two counters vectors $s_{i}$ and $r_{i}$ such that :

$\forall j \neq i: s_{i}[j]=\sharp m_{i j}$ sent over $c_{i j}$ since last visit 
$\forall j \neq i: r_{i}[j]=\sharp m_{j i}$ received on $c_{j i}$ since last visit

(where $\sharp \cdots$ denotes "the number of ...")

$s_{i}[j]$ contributes to $M T(\mu)[j](j \neq i)$ and $\sum_{j \neq i} r_{i}[j]$ to $M T(\mu)[i]$ when the event visit $(i, \mu)$ occurs.

Let's denote $s_{i}(\mu)[j], r_{i}(\mu)[j]$ the value of counter at time $r_{i}(\mu)$. The wave algorithm can now be stated :

For every wave $\mu$ :

wave $\mu$ carries over a counter vector $C O L L$ and a boolean $T L$

$\rightarrow$ Initially (wave $\mu$ )

$\operatorname{COLL}(\mu) \leftarrow \operatorname{COLL}(\mu-1)$

$\rightarrow$ Upon visit $(i, \mu)$

$\forall j \neq i \operatorname{COLL}(\mu)[j] \leftarrow \operatorname{COLL}(\mu)[j]+s_{i}(\mu)[j]$

$\operatorname{COLL}(\mu)[i] \leftarrow \operatorname{COLL}(\mu)[i]-\sum_{j \neq i} r_{i}(\mu)[j]$

reset to 0 counters $s_{i}, r_{i}$;

if $T L$ then save last recorded state $L S_{i}^{(\mu-1)}$ endif ;

record $L S_{i}^{(\mu)}$

$\rightarrow$ Upon return $(\mu)$

$\% \forall i C O L L(\mu)[i]$ contains the number of intransit messages

towards $P_{i}$ in $G S(\mu) \%$

$T L \leftarrow \operatorname{COLL}(\mu) \equiv 0$

The result "GS( $\mu)$ is transitless" is diffused by wave $\mu+1$ (through boolean $T L$ ) : liveness delay is equal to 1 wave.

\section{2 non FIFO communication}

\subsubsection{Abstract global definitions}

Consider the following global abstract quantities :

$$
\begin{aligned}
S(\mu) & =\sharp(\text { messages sent in } G S(\mu)) \\
R(\mu) & =\sharp(\text { messages received in } G S(\mu)) \\
N T(\mu) & =\sharp(\text { intransit messages in } G S(\mu)) \\
N(\mu) & =\sum_{\nu \leq \mu} S(\nu)-\sum_{\nu \leq \mu-1} R(\nu)
\end{aligned}
$$


We have :

$$
N(\mu)=\sum_{\nu \leq \mu-1} S(\nu)-\sum_{\nu \leq \mu-1} R(\nu)+S(\mu)
$$

whence

$N(\mu)=N T(\mu-1)+S(\mu)$

Moreover, the following relation is straightforward from the definitions :

$$
N T(\mu)=N(\mu)-R(\mu)
$$

\subsubsection{Distributed implementation}

Each controller $C T L_{i}$ is endowed with two counters :

$s_{i}=$ total number of messages sent since the last time local state has been recorded

$r_{i}=$ total number of messages received since the last time local state has been recorded (actually, on each incoming channel, up to marker reception : cf. receptions freezing).

Moreover, upon each local state recording, $C T L_{i}$ resets $s_{i}, r_{i}$ to 0 . Values obtained at time $\tau_{i}(\mu)$ will be denoted $s_{i}(\mu), r_{i}(\mu)$.

Wave $\mu$.performs the following tasks :

- saves $N T(\mu-1)$ as $N T B A C K$ (computed by wave $\mu-1)$,

- computes sums $S(\mu)=\sum_{i} s_{i}(\mu), R(\mu)=\sum_{i} r_{i}(\mu)$.

Upon return, the following computations can be performed :

$$
\begin{array}{ll}
N(\mu) & \leftarrow N T B A C K+S(\mu) \\
N T(\mu) & \leftarrow N(\mu)-R(\mu) \\
N T B A C K & \leftarrow N T(\mu)
\end{array}
$$

Boolean information "GS( $\mu)$ is transitless" is equal to $N T(\mu)=0$ and can be diffused by wave $\mu+1$. Thus, liveness delay of 1 wave is necessary, as in the FIFO case; size of data carried over by a wave is equal to

- two integers (sums $E, R$ ), 
- possibly one integer (NTBACK) if, due to the wave implementation, this result cannot be stored on a particular node,

- possibly one boolean $T L=(N T B A C K=0)$ if $N T B A C K$ is not carried over by wave.

\section{Conclusion}

Observing consistent global states is a fundamental problem in distributed systems, and a dificult one in asynchronous models. We gave a clear definition of global states, bringing out distinction between observable (processes) and not observable (channels) local states, and some of their properties (consistency, being transitless). The problem was tackled by steps : at first level, it was shown how a process can record its own local state consistently with its neighbours; the technique of markers, similar in the FIFO case to the one developped in [CL85, CM86], is generalized, through acknow- ledgements, to the non FIFO communication model. At second level, global synchronization requiring the wave sequence abstract control scheme solves the problem stated; from a methodological point of view, it displays yet another situation where this abstract network traversal scheme can be seen as a syntaxic scanner on which semantic actions are grafted : this approach has been previously enhanced in [HR88a]. Finally, at third level, a computation model is presented through an example : computing the number of intransit messages; clearly, other functions fit this model, e.g. set of intransit messages, or - in a bounced FIFO comunication context - number of lost messages due to full buffer (assuming that control messages have a specific storage buffer preventing them from loss), etc..

As conclusion, we lay stress on the fact that techniques developped lead to a whole family of algorithms devoted to observation of asynchronous distributed applications or systems.

\section{References}

[CL85] K. M. Chandy and L. Lamport. Distributed snapshots: determining global states of distributed systems. ACM TOCS, 63-75, February 1985. 
[CM86] K. M. Chandy and J. Misra. An example of stepwise refinement of distributed programs: quiescence detection. ACM TOPLAS, $8(3)$, July 1986.

[CM88] K. M. Chandy and J. Misra. Parallel program design : a foundation. Addison-Wesley, 1988.

[FGL82] M. J. Fischer, N. D. Griffeth, and N. Lynch. Global states of a distributed system. IEEE trans. on soft. eng., SE-8:3:198-202, may 1982 .

[HJPR87] J.-M. Hélary, C. Jard, N. Plouzeau, and M. Raynal. Detection of stable properties in distributed applications. ${ }^{\text {th }} A C M$ SIGACTSIGOPS, Symp. Principles of Distributed Computing, Vancouver, Canada, 125-136, August 1987.

[HPR89] J.M. Hélary, N. Plouzeau, and M. Raynal. A characterization of a particular class of distributed snapshots. In Submitted to International Conference on Computing and Information (ICCI'89), Toronto, may 23-27 1989.

[HR88a] J.M. Hélary and M. Raynal. Les parcours distribués de réseaux: un outil pour la conception de protocoles. In $\mathrm{R}$. Castanet et $\mathrm{O}$. Rafiq, editor, CFIP'88 Ingénierie des protocoles, pages 159170, Eyrolles, 1988.

[HR88b] J.-M. Hélary and M. Raynal. Synchronisation et contrôle des systèmes et des programmes répartis. Eyrolles, Septembre 1988. English translation to appear, Wiley, 1990.

[HR89] J.-M. Hélary and M. Raynal. An abstract distributed iteration scheme: application to the computation of weighted shortest paths. Technology and Science of Informaiics, Vol. 7, No צ, May 1989. (In French).

[Lam78] L. Lamport. Time, clocks and the ordering of events in a distributed system. Communications. of the ACM, 21(7):558-565, July 1978.

[LY87] T.H. Lai and T.H Yang. On distributed snapshots. Inf. Proc. Letters, 25:153-158, 1987. 
[Mat89] F. Mattern. Virtual time and global states of distributed systems. In Cosnard, Quinton, Raynal, and Robert, editors, Proc. Int. Workshop on Parallel and Distributed Algorithms, Bonas, France, oct. 1988, North Holland, 1989.

[Mor85] C. Morgan. Global and logical time in distributed algorithms. Inf. Proc. Letters, 20:290-294, 1985.

[Sch85] F. B. Schneider. Paradigms for distributed programs. In Distributed Systems, pages 431-480, Springer Verlag, 1985. LNCS 190.

[Tel86] G. Tel. Distributed Infimum Approximation. Tech. report RUU. CS-86-12, University of Utrecht, 1986. 
PI 460 FLOW ANALYSIS IN TANDEM QUEUES WITH FEEDFORWARD FLOWS

Kamel SISMAIL

14 Pages, Février 1989.

PI 461 NUMERICAL CONCERNS IN CONVOLUTION-TYPE ALGORITHMS Gerardo RUBINO, William STEWART 18 Pages, Février 1989.

PI 462 ACCUMULATED REWARD OVER THE N FIRST OPERATIONAL PERIODS IN FAULT-TOLERANT COMPUTING SYSTEMS Gerardo RUBINO, Bruno SERICOLA 14 Pages, Mars 1989.

PI 463 ELEMENTS FINIS $C^{1}$, POLYNOMIAUX DE DEGRE QUATRE PAR TRIANGLE, DANS UNE TRIANGULATION FORMEE DE TRIANGLES EQUILATERAUX

Michel CROUZEIX, Miloud SADKANE 26 Pages, Mars 1989.

PI 464 VERS UN MODELE D'ECLAIREMENT REALISTE Pierre TELLIER, Kadi BOUATOUCH 66 Pages, Avril 1989.

PI 465 COMPILATION OF FUNCTIONAL LANGUAGES BY PROGRAM TRANSFORMATION

Pascal FRADET, Daniel LE METAYER 28 Pages, Avril 1989.

PI 466 MODEL BASED DIAGNOSIS : A CASE STUDY IN VIBRATION MECHANICS

Michèle BASSEVILLE, Albert BENVENISTE 26 Pages, Avril 1989.

PI 467 DELAI DE COMMUNICATION ENTRE NOEUDS VOISINS SUR L'iPSC/2

Patrice BURGEVIN, André COUVERT, René PEDRONO 16 Pages, Avril 1989.

PI 468 OBSERVING GLOBAL STATES OF ASYNCHRONOUS DISTRIBUTED Jean-Michel HELARY

24 Pages, Avril 1989.

$$
\begin{gathered}
\text { Imprimé en France } \\
\text { par } \\
\text { l'Institut National de Recherche en Informatique et en Automatique }
\end{gathered}
$$


